\title{
Reproductive Decision-Making in a Macro-Micro Perspective
}

\author{
edited by D. Philipov, A.C. Liefbroer, and J. Klobas \\ Dordrecht: Springer, 2015 \\ ISBN 978-94-017-9400-8 \\ Hardcover or softcover $103.99 €, 178$ pp.
}

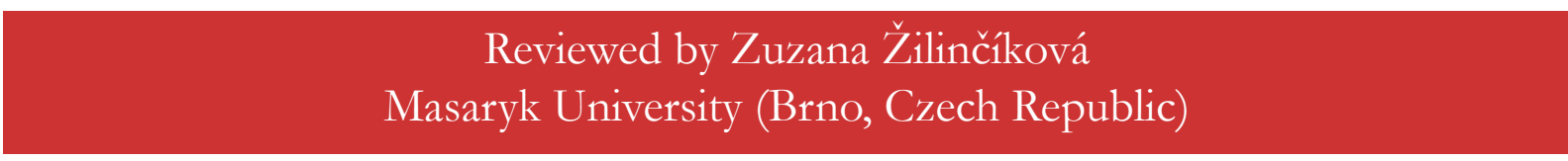

Reproductive Decision-Making in a Macro-Micro Perspective is one of the outcomes of a European Commissionfunded project of the same name (abbreviated REPRO). The project, realized between 2008 and 2011, focuses on the discrepancies between intended and actual fertility. Although the book does not intend to summarize the project, it provides a compelling collection of the individual outcomes, and it offers a snapshot of the project's results. As in the project, the so-called fertility gap is central to the content of this edited volume. The authors take both thematically and methodologically different perspectives to examine how intentions are formed, and how they are translated into behaviour. The topic is timely, given the persistent low fertility rates in many European countries, but the theme has also a universal application as it addresses the fundamentals of the processes of intention formation and realization.

The common framework underlying the book is the Theory of Planned Behaviour, a general concept developed in social psychology by Ajzen (1988). TPB, designed for the study of reasoned behaviour, posits that intention formation is determined by three components: attitudes, subjective norms, and perceived behavioural control. The intentions are then translated into behaviour that is conditional on the actual behavioural control. Further, all chapters share an interest in the micro-macro link. The authors investigate the embeddedness of the individual's intention formation and the translation to behaviour (micro-level) in the wider societal context (macro-level). Indeed, all the data sources have a cross-national character, with a focus on European countries. Notably, they include Central and Eastern European countries, which are often neglected in other studies.

The application of the Theory of Planned Behaviour to fertility behaviour presented in the book is performed with a great deal of thoughtfulness and reflexivity. Chapters 3 and 5 each explore the formation of fertility intentions from different angles. Through quantitative analysis, Chapter 3 finds that the importance of individual TPB components is dependent on contextual factors, such as individual life-course stage or country. Chapter 5 studies the formation of fertility intention through an analysis of semi-structured interviews. Stepping away a little from the TPB framework, the authors point to the role of desires and uncertainty in the intention formation process. The analysis reveals that while desire underlies the intention to have a child in a given timeframe, uncertainties are crucial in explaining the discrepancies between intentions and their realization. A challenge to TPB is also present in Chapter 4, which shows that the realization of fertility intentions is influenced by societal context; generally, TPB does not account for such a link. In sum, the critical application of the TPB framework presented by the authors of the book shows that it functions relative to individual and societal contexts, and that the TPB does not necessarily account for all the influences in the process of intention formation and realization.

One of the most valuable contributions of the book is its exploration of how societal context influences the formation and realization of fertility intention. For example, Chapter 2 shows how family policies, including financial transfers such as leave entitlements and child-care services, influence fertility on a macro level. The comprehensive analysis compares these family policies across a variety of countries and relates it to fertility 
outcomes. In Chapter 6, the authors show how a macro-level factor-a country's advancement in the Second Demographic Transition - influences individual norms regarding fertility behaviour, such as voluntary childlessness or childbearing outside of marriage. The book thus shows that it is crucial to consider the societal context in which the fertility decision-making takes place and that researchers and policymakers should be cautious about unwary generalization of country-specific findings.

It is difficult to find critiques regarding such an elaborate piece of scholarship, with its well-crafted individual chapters. One would only wish to read more about the REPRO outcomes. Perhaps the only disadvantage of the collection is the lack of connection between the chapters. Although the introduction and conclusion do their best in stressing the common points of the book, it reads as though the chapters were put together ad hoc. Nevertheless, the thematic variation of the chapters makes the book stimulating, and the final chapter, which comments on the other REPRO outcomes, helps to put the findings of the individual chapters into the broader context of the project.

This book is appealing for all who are curious about fertility, family planning, and family policy. The chapters, along with the other outcomes of the REPRO project, systematically study fertility decision formation and realization. It is, therefore, a valuable source of knowledge and inspiration for other researchers or more advanced students of demography, sociology, and population sciences. Policymakers can also benefit from reading the book, as it increases understanding of how to help people realize their fertility plans, and thus to potentially increase low fertility.

\section{Reference}

Ajzen, I. 1988. Attitudes, Personality, and Behavior. Berkshire and New York: Open University Press. 